\title{
Successful satellite lift-off
}

\section{Washington}

Aт 15 tonnes, the Gamma Ray Observatory (GRO) is the heaviest scientific satellite ever carried by the Space Shuttle. It was launched last Friday (5 April) and deployed two days later from the cargo bay of space shuttle Atlantis into Earth orbit, where it joins the Hubble Space Telescope as the second of four 'Great Observatories' planned by the National Aeronautics and Space Administration (NASA). The third and fourth Great Observatories are the Advanced X-ray Astronomy Facility (AXAF), tentatively scheduled for launch in 1998, and the Space Infrared Telescope Facility (SIRTF), not yet approved but endorsed by the recent Bahcall committee report as the highest priority among big astronomy projects (see Nature 350, 185; 21 March 1991).

GRO comprises four separ-

Preparing for launch - the observatory undergoes tests. ate instruments, each designed to study the gamma-ray sky with different spatial resolutions and in different energy ranges. The unusual weight of GRO is due to two of its instruments in particular, EGRET (Energetic Gamma Ray Experiment Telescope) and COMPTEL (Imaging Compton Telescope), GERMAN RESEARCH

\section{Berlin finally licenses}

\section{reactor}

\section{Munich}

Wrrt the recent licensing of the refurbished reactor at the Hahn-Meitner Institute (HMI) in Berlin, German and foreign researchers can at last begin to use a nuclear reactor that serves primarily as a neutron source. The licence was announced by the newly elected Berlin government on 26 March. The reactor will begin a testing phase this week, and should be available for experiments by the end of the year.

The decisive factor in licensing the reactor was the resurgence in Berlin of the conservative Christian Democratic Union or CDU. CDU, which made licensing the reactor a high priority, received the most votes in elections on 2 December 1990.

The licensing had been delayed for nearly two years because of opposition from the local Green Party, known as the Alternative Liste. In 1989, the Greens halted the licensing procedure, begun in 1979 , as it neared a conclusion. They objected to licensing the $10-\mathrm{MW}$ reactor on the grounds that no suitable arrangements had been made to dispose of the waste it generated (see Nature 346, 688; 1990).

Steven Dickman both of them the product of US-European collaborations. Because gamma rays cannot be focused by refracting devices such as optical lenses, imaging in these telescopes is accomplished by means of 'grazing incidence' reflectors, in which gamma rays are made to skate almost tangentially across a smooth metal surface, being deflected just

\section{IMAGE UNAVAILABLE FOR COPYRIGHT REASONS}

enough to bring them to a focus a metre or so away. To produce a useful photon collection area, a series of slightly conical cylinders is assembled concentrically to form a massive gamma-ray 'lens'.

For the first 15 months of GRO's mission, both EGRET and COMPTEL will be devoted largely to sky-survey work. More sensitive by a factor of ten than any previous gamma-ray detectors, they are likely to trawl up large numbers of new astrophysical gamma sources, as well as measuring accurately the cosmologically important gammaray background.

Completing GRO's line-up are the Oriented Scintillation Spectrometer Experiment (OSSE) and the Burst and Transient Source Experiment (BATSE). OSSE will measure gamma-ray spectra in the energy range $100 \mathrm{keV}$ to $10 \mathrm{MeV}$, where characteristic line emission from nuclear processes and radioactivity is produced. Its main observational targets will be astrophysical objects such as supernovae and novae, where heavy elements are manufactured, and pulsars and X-ray binaries, which generate electrons and photons energetic enough to induce nuclear activity.

BATSE consists of eight identical instruments mounted at GRO's corners and designed to watch the sky constantly for gamma-ray bursts. First observed two decades ago, gamma-ray bursts have been catalogued by the hundred but remain largely unexplained; they occur sporadically and unpredictably, and have not been conclusively associated with any known astrophysical object. BATSE's eight detectors can see almost the whole sky, and when a burst is seen by one of the units, as many as three others can quickly be trained in the same direction.

David Lindley

\section{Ozone loss worse than expected}

\section{Washington}

THE protective ozone in the Earth's atmosphere is disappearing much faster than previously thought, according to a study done by researchers at the National Aeronautics and Space Administration (NASA). The results, which have been reviewed at a workshop but not yet submitted for publication, were announced last week by William Reilly, director of the Environmental Protection Agency.

"These data suggest depletion of four to five per cent has occurred since 1978 over the United States," Reilly said. "Past studies had shown about half that amount." The ozone depletion is widely believed to be caused by the release into the atmosphere of chlorofluorocarbons (CFCs), man-made gases used for refrigeration, the manufacture of insulating foams and as cleaning solvents in making computer chips.

Besides the larger-than-expected drop, ozone loss has been found further south than previously seen. And more importantly, the ozone depletion has been detected well into the spring months, whereas researchers had previously believed that ozone depletion was significant only in winter. (Warmer temperatures are believed to slow down the chemical reactions by which chlorine from the CFCs breaks down the ozone.) Because people are more likely to be outside and exposed to the Sun in the warmer months, and because scientists estimate that for every one per cent decrease in upper atmosphere ozone an extra two per cent of the Sun's ultraviolet radiation reaches the Earth's surface, the springtime loss threatens to increase the incidence of skin cancer. Reilly said that the ozone depletion could nearly double the skin cancer rate over the next $\mathbf{5 0}$ years, causing as many as 200,000 extra deaths in the United States alone.

The data on which these conclusions are based were taken by the 'total ozone mapping spectrometer', an instrument carried on a NASA satellite. The instrument measures 'column ozone' - the amount of ozone in the atmosphere above a given spot on the Earth's surface. The data were analysed by Richard Stolarsky, an atmospheric scientist at NASA's Goddard Space Flight Center in Greenbelt, Maryland.

The ozone depletion extends across much of the Northern Hemisphere above a latitude of 30-35 degrees, including most of the United States, Europe and Asia. It may bolster demands to phase out the production and use of CFCs even more quickly than agreed in the 1987 Montreal Protocol, which calls for industrial nations to cease production by 2000 and less developed nations by 2010 .

Robert Pool 